\title{
A Randomized, Placebo-Controlled Trial of Gamma Linolenic Acid as an Add-on Therapy to Minocycline for the Treatment of Rosacea
}

\author{
Ji Hyun Kim, Yong Woo Oh, Dong Hee Kim, Byeong Hak Seo, Ho Seok Suh, Yu Sung Choi \\ Department of Dermatology, Ulsan University Hospital, University of Ulsan College of Medicine, Ulsan, Korea
}

Background: A recent study suggested a possible role of skin barrier dysfunction in the pathogenesis of rosacea, which leads to irritation symptoms. Gamma linolenic acid (GLA) is an essential omega- 6 fatty acid that is known to restore defective epidermal skin barrier. GLA supplementation has not previously been performed in rosacea patients. Objective: To investigate the efficacy and safety of adding GLA to minocycline compared to minocycline alone in rosacea patients. Methods: This prospective, double-blind, randomized, placebo-controlled trial enrolled 31 rosacea patients. They were randomly assigned to receive $320 \mathrm{mg} /$ day of GLA (Evoprim ${ }^{\circledR}$ ) $(n=16)$ or placebo $(n=15)$ in addition to $100 \mathrm{mg} /$ day of minocycline for 8 weeks. Investigator's global assessment (IGA) and patient's global assessment (PGA) were used to assess clinical severity at weeks $0,4,8$, and 12 . Biophysical parameters including melanin index, erythema index, transepidermal water loss (TEWL), lipid concentration, and stratum corneum hydration were measured. Results: In the GLA group, a higher proportion of patients achieved treatment success $($ IGA $\leq 1)$ at week $8(68.75 \%$ vs. $33.33 \%)$ and patient satisfaction (PGA $\geq 3)$ at weeks $8(75.0 \%$ vs. $40.0 \%)$ and $12(81.3 \%$ vs. $46.6 \%$ ). Both groups, throughout 12 weeks of treatment,

Received November 18, 2019, Revised February 24, 2020, Accepted for publication May 25, 2020

Corresponding author: Yu Sung Choi, Department of Dermatology, Ulsan University Hospital, University of Ulsan College of Medicine, 877 Bangeojinsunhwando-ro, Dong-gu, Ulsan 44033, Korea. Tel: 82-52-250-7090, Fax: 82-52-250-7155, E-mail: uuhderma@hanmail.net ORCID: https://orcid.org/0000-0001-8308-4091

This is an Open Access article distributed under the terms of the Creative Commons Attribution Non-Commercial License (http://creativecommons. org/licenses/by-nc/4.0) which permits unrestricted non-commercial use, distribution, and reproduction in any medium, provided the original work is properly cited.

Copyright (c) The Korean Dermatological Association and The Korean Society for Investigative Dermatology revealed a trend toward improvement in erythema index, melanin index, TEWL, and stratum corneum hydration. Particularly, there was a significant difference in TEWL and stratum corneum hydration over time between the two groups ( $p=0.033, p=0.003$, respectively). No serious adverse event was observed in both groups. Conclusion: GLA is beneficial as an additional therapeutic option for rosacea patients treated with minocycline. (Ann Dermatol 32(6) $466 \sim 472,2020$ )

\section{-Keywords-}

Gamma-linolenic acid, Minocycline, Rosacea

\section{INTRODUCTION}

Rosacea is an inflammatory skin disease, which is characterized by erythema, flushing, with or without papules or pustules, and telangiectasias ${ }^{1}$. In addition, many rosacea patients experience other symptoms such as burning, drying, and itching which can considerably impair quality of life and cause cosmetic problems ${ }^{2}$.

Its pathophysiology is not completely elucidated and thought to be multifactorial ${ }^{3}$. Recent studies highlighted the skin barrier abnormalities in rosacea ${ }^{4}$, which induces chronic perivascular, dermal, and epidermal inflammation. This chronic inflammation leads to increased transepidermal water loss (TEWL) and decreased stratum corneum hydration, thus initiating a vicious cycle.

Various treatment modalities are attempted for rosacea, given its complex pathogenesis. Particularly, tetracycline antibiotics such as doxycycline and minocycline have been successfully used in the treatment of rosacea, inhibiting various pathways of inflammation ${ }^{5}$. However, due to the chronic nature of the disease, long-term therapy results in 
increased possibility of dose-related adverse events (AEs), such as hepatitis ${ }^{6}$ and hyperpigmentation ${ }^{7}$. Furthermore, because of skin barrier deficiency and vascular hyperreactivity, patients frequently experience irritation symptoms such as erythema, stinging, burning, and dryness ${ }^{8}$.

Evening primrose oil (EPO) is reported to restore a defective epidermal skin barrier, to normalize excessive TEWL, and to improve skin barrier in healthy people ${ }^{9,10}$ and in patients with atopic dermatitis ${ }^{11}$, acne vulgaris patients treated with isotretinoin ${ }^{12}$, and xerosis ${ }^{13}$ after systemic administration.

We hypothesized that systemic administration of gamma linolenic acid (GLA) can improve signs and symptoms of rosacea by restoring skin barrier function. Therefore, in this study, we aimed to investigate the efficacy and safety of GLA as an add-on to minocycline compared to minocycline alone in rosacea patients.

\section{MATERIALS AND METHODS}

The study was conducted as a prospective, double-blind, randomized, placebo-controlled trial. The study protocol was approved by the Institutional Review Board of Ulsan University Hospital (IRB no. 2017-03-038), and written informed consent was obtained from all subjects.

We received the patient's consent form about publishing all photographic materials.

\section{Participants}

In this study, 31 patients with rosacea aged 18 years and above were equally randomized into groups $A$ and $B$ by block randomization with block size of 2 . Subjects with following conditions were excluded: having another uncontrolled skin disease or systemic disease, having a present or prior history of atopic dermatitis, pregnant or planning pregnancy, having known hypersensitivity to tetracyclines, having or suspected to have hepatic impairment, and received systemic treatment within a month.

\section{Randomization and intervention}

Enrolled patients were randomly assigned in a 1:1 ratio to the experimental and control group by block randomization with block size of 2 . Randomization codes were given to the study products so that the clinical investigator and subjects cannot recognize them.

During the 8-week treatment period, the experimental group received $320 \mathrm{mg}$ per day of GLA with minocycline $50 \mathrm{mg}$ twice daily. The control group received placebo capsules with minocycline $50 \mathrm{mg}$ twice daily. Each active capsule contained $450 \mathrm{mg}$ of EPO and $40 \mathrm{mg}$ of GLA, while the placebo capsule contained $480 \mathrm{mg}$ of soybean oil. Active and placebo capsules were donated by Dalim ${ }^{\circledR}$ (Seoul, Korea) (Fig. 1).

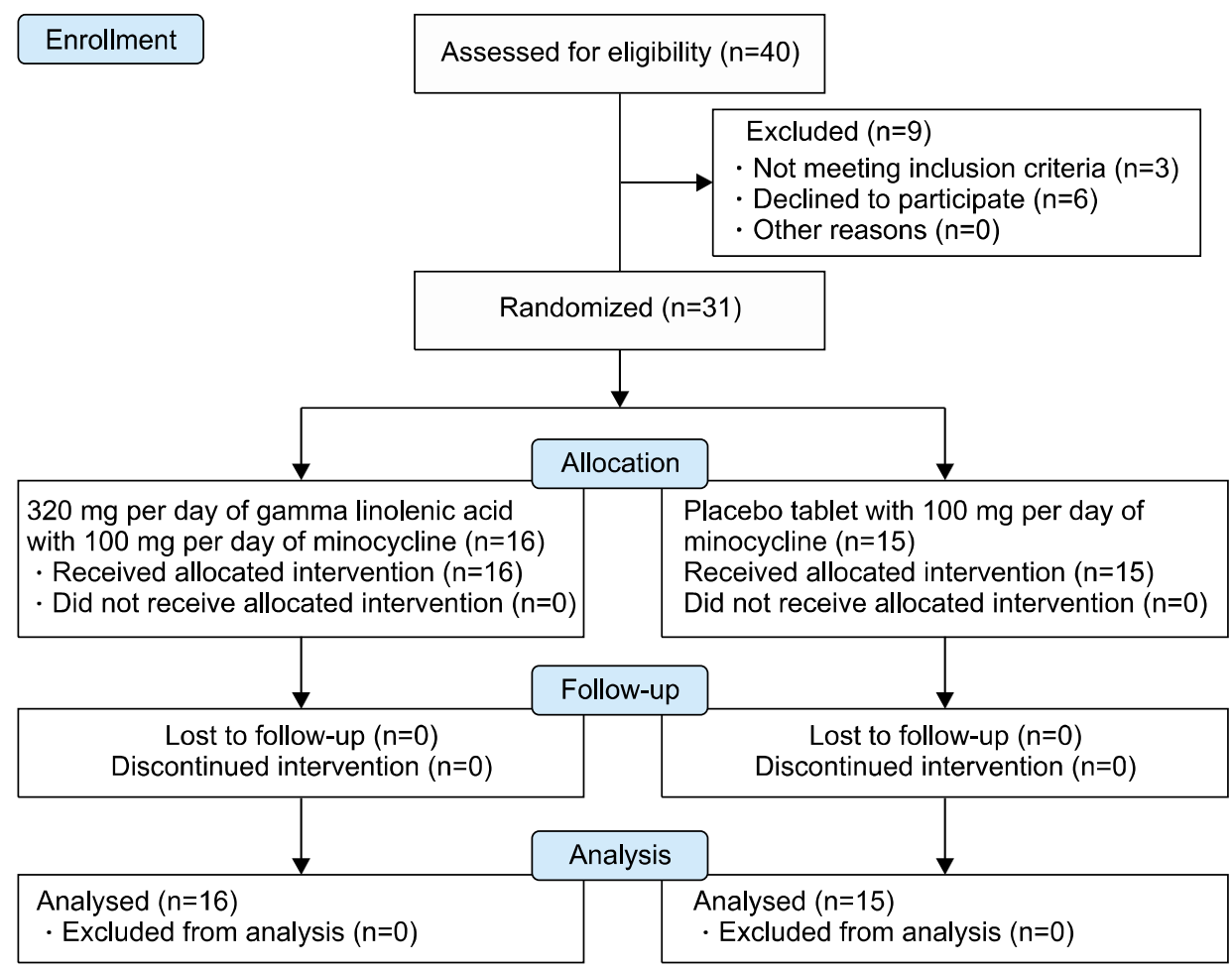

Fig. 1. Flow chart of the trial. 


\section{Assessment}

Patients visited the outpatient clinic at screening, during treatment (weeks 0, 4, and 8), and follow-up after treatment (week 12). At each visit, clinical photography was performed. Clinical severity was assessed by investigator's global assessment (IGA) score and patient's global assessment (PGA) score. The IGA score was recorded using a 5-point scale: 0 (clear), 1 (minimal), 2 (mild), 3 (moderate), and 4 (severe). PGA was recorded using a 5-point scale: 0 (poor), 1 (fair), 2 (good), 3 (very good), and 4 (excellent). AEs were monitored at each visit.

Biophysical parameters indicating melanin index, erythema index, TEWL, lipid concentration, and stratum corneum hydration were measured by Mexameter ${ }^{\circledR}$ (Courage \& Khazaka, Cologne, Germany), Tewameter ${ }^{\mathbb{R}}$ (Courage \& Khazaka) Sebumeter ${ }^{\mathbb{R}}$ (Courage \& Khazaka), and Corneometer $^{\circledR}$ (Courage \& Khazaka), respectively. Patients were directed to use the same skin care products and to rest for 15 minutes in the same allocated room with room temperature of $24^{\circ} \mathrm{C} \sim 25^{\circ} \mathrm{C}$ and humidity of $18 \% \sim 24 \%$ before the measurement. Each parameter was repeatedly measured 4 times at the perinasal cheek, and the mean value was recorded.

\section{Statistical analysis}

The demographic differences between the two treatment groups were analyzed by using Mann-Whitney $U$ test and Pearson's chi-square test. Pearson's chi-square test was used for comparing treatment success rate according to the IGA and PGA scores between the placebo and GLA group. Repeated measures analysis of variance was used for statistical analysis of biophysical parameters. Statistical analysis was performed using IBM SPSS statistics ver. 24.0 (IBM Corp., Armonk, NY, USA). A p-value of $<0.05$ was considered statistically significant.

\section{RESULTS}

\section{Patient demographics}

In this study, 31 rosacea patients aged 18 years or older who visited the outpatient clinic between May 2017 and March 2018 were enrolled. The mean age of patients who completed the study was 47.7 years (range, $24 \sim 80$ years). Of the 31 patients, 20 were female $(64.5 \%)$ and 11 were male $(35.5 \%)$. The mean disease duration was 28.35 months (range, 5 49 months). Both erythematotelangiectatic rosacea (ETR) and papulopustular rosacea (PPR) subtypes were included. The GLA group was composed of eight patients with ETR and eight patients with PPR, while the placebo group was composed of eight patients with ETR and seven patients with PPR. No significant difference in patient characteristics was found between the GLA and placebo groups (Table 1).

\section{Treatment success (investigator's global assessment $\leq 1$ )}

Treatment success was defined as IGA score of 0 (clear) or 1 (minimal). At week 8, the proportion of patients who achieved treatment success was significantly higher in the GLA group than in the placebo group $(68.75 \%$ vs. $33.33 \%$, $p=0.049$ ). At week 12 , the proportion of patients who achieved treatment success was higher in the GLA group but without significant difference $(56.25 \%$ vs. $26.67 \%$, $p=0.095$ ) (Fig. 2A). Selected clinical photographs showing the changes of patients in each group over the course of the study (Fig. 3) shows a patient in the GLA group showed decreased erythema and numbers of papules at weeks 8 and the improvement was well maintained up to weeks 12 . However, a patient in the placebo group also showed decreased number of papules, but few papules and background erythema remained at week 8 and week 12. Furthermore, she complained of stinging and dryness until the end of the treatment.

\section{Patient satisfaction (patient's global assessment $\geq 3$ )}

Patient satisfaction was defined as PGA score of 3 (very good) or 4 (excellent). At 8 and 12 weeks, the proportion of patients achieving PGA score of 3 (very good) or 4 (excellent) were $75.0 \%$ and $81.3 \%$ for the GLA group and $40.0 \%$ and $46.6 \%$ for the placebo group, respectively. Statistical analyses showed that patients who received GLA were more likely to achieve a PGA score of 3 or 4 at 8 and 12 weeks compared with patients who received placebo ( $p=0.048, p=0.044$, respectively) (Fig. 2B).

Table 1. Demographics of patients

\begin{tabular}{lcccc}
\hline $\begin{array}{c}\text { Character- } \\
\text { istic }\end{array}$ & $\begin{array}{c}\text { GLA group } \\
(\mathrm{n}=16)\end{array}$ & $\begin{array}{c}\text { Placebo group } \\
(\mathrm{n}=15)\end{array}$ & $\begin{array}{c}\text { Total } \\
(\mathrm{n}=31)\end{array}$ & $p$-value \\
\hline Age $(\mathrm{yr})$ & $48.18 \pm 14.77$ & $47.2 \pm 13.16$ & $47.70 \pm 13.79$ & $0.8^{*}$ \\
Sex & & & & $0.81^{\dagger}$ \\
$\quad$ Male & $6(37.5)$ & $5(33.3)$ & $11(35.5)$ & \\
$\quad$ Female & $10(62.5)$ & $10(66.7)$ & $20(64.5)$ & \\
$\begin{array}{c}\text { Disease } \\
\text { duration }\end{array}$ & $25.93 \pm 15.24$ & $30.93 \pm 10.72$ & $28.35 \pm 13.28$ & $0.38^{*}$ \\
(mo) & & & & \\
Subtype & & & & \\
$\quad$ PPR & $8(50.0)$ & $7(46.7)$ & $15(48.4)$ & \\
$\quad$ ETR & $8(50.0)$ & $8(53.3)$ & $16(51.6)$ & \\
\hline
\end{tabular}

Values are presented as mean \pm standard deviation, number (\%), or number only. GLA: gamma linolenic acid, PPR: papulopustular rosacea, ETR: erythematotelangiectatic rosacea. *Mann-Whitney U-test, ${ }^{\dagger}$ Pearson chi-square test. 
A

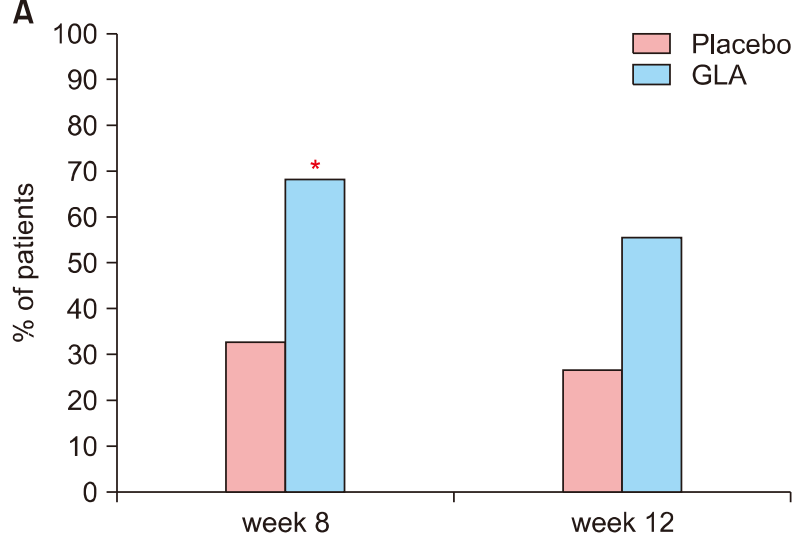

B

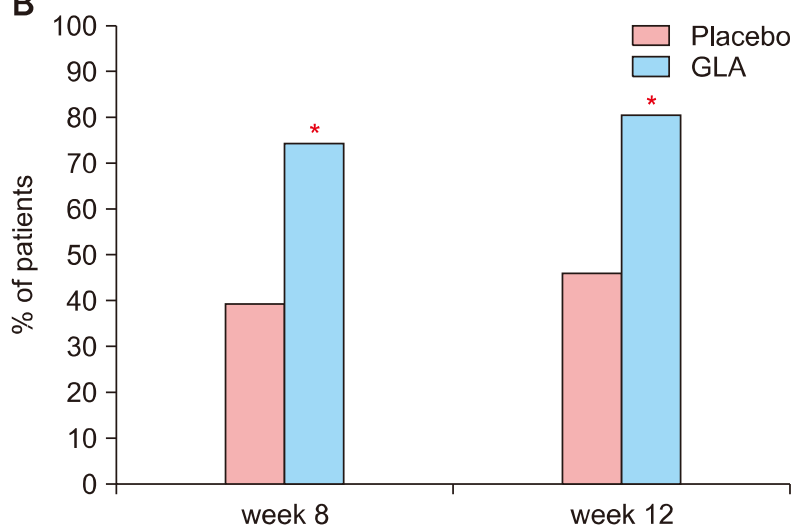

Fig. 2. Clinical efficacy outcomes over 12 weeks. (A) Proportion of patients achieving treatment success (investigator's global assessment $[\mathrm{IGA}] \leq 1$ ). (B) Proportion of patients achieving treatment satisfaction (patient's global assessment [PGA] $\geq 3$ ). GLA: gamma-linolenic acid. *Statistically significance $(p<0.05)$.

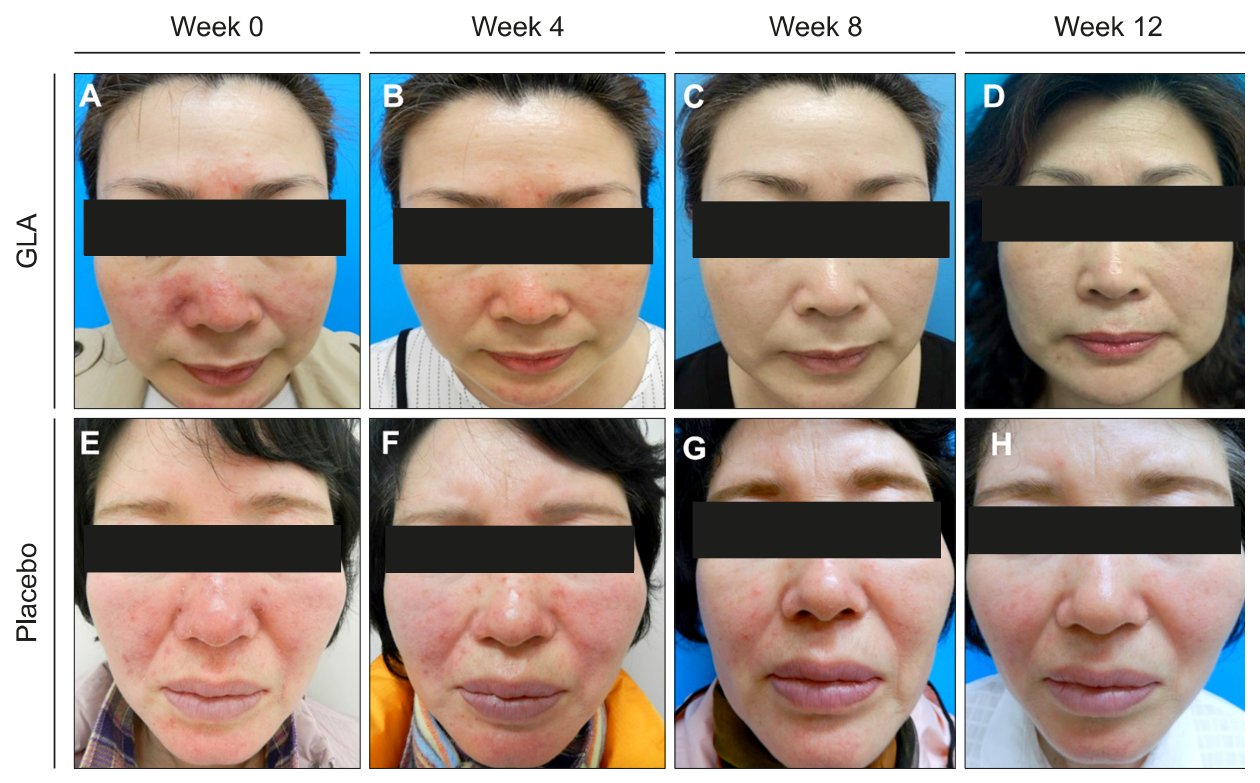

Fig. 3. Representative clinical photographs from patients in different randomized group showing clinical improvement of 12 weeks of treatment course. $(\mathrm{A} \sim \mathrm{D}) \mathrm{A}$ 45-year-old female patient in the gamma-linolenic acid (GLA) group showed significant improvement at weeks 8 and well maintained up to weeks 12. $(E \sim H)$ A 56-year-old female patient in the placebo group. At week 8 , reduction in numbers of papules and pustules were observed, but background erythema remained present. At week 12, aggravation of erythema is observed.

\section{Biophysical parameters}

The mean baseline erythema index in the placebo and GLA groups were $414.73 \pm 158.50$ au and $426.75 \pm 161.08$ $\mathrm{au}$, respectively, and the mean baseline melanin index was $135.85 \pm 34.71$ au and $142.50 \pm 67.47$ au, respectively. After 8 weeks of treatment, the mean erythema index in the placebo and GLA groups were decreased to $379.93 \pm$ 131.84 au and $354.86 \pm 147.95$ au, which was well maintained to week 12 at $363.60 \pm 118.08$ au and $342.75 \pm$ 145.94 au, respectively (Fig. 4A). The mean melanin index decreased over time in the placebo and GLA groups at week 8 with $127.67 \pm 29.50$ au and $122.04 \pm 53.60$ au. It was also sustained to week 12 at $125.73 \pm 35.28$ au and $122.31 \pm 48.00 \mathrm{au}$, respectively (Fig. 4B).

The mean TEWL in the placebo group was $18.35 \pm 4.06$ $\mathrm{g} / \mathrm{h} / \mathrm{m}^{2}$ at baseline, $14.95 \pm 4.32 \mathrm{~g} / \mathrm{h} / \mathrm{m}^{2}$ at week 8 , and $14.58 \pm 5.48 \mathrm{~g} / \mathrm{h} / \mathrm{m}^{2}$ at week 12 , while the mean TEWL in the GLA group was $18.07 \pm 6.40 \mathrm{~g} / \mathrm{h} / \mathrm{m}^{2}$ at baseline, $10.48 \pm$ $3.19 \mathrm{~g} / \mathrm{h} / \mathrm{m}^{2}$ at week 8 , and $9.07 \pm 2.75 \mathrm{~g} / \mathrm{h} / \mathrm{m}^{2}$ at week 12 . TEWL showed greater improvement in the GLA group compared to placebo group ( $p=0.033$ ) (Fig. 4C).

Furthermore, the mean stratum corneum hydration status (corneometer index) in the placebo and GLA groups were $36.28 \pm 8.14$ au and $36.18 \pm 8.72$ au at baseline, which increased to $44.20 \pm 8.87$ au and $54.85 \pm 9.31$ au at week 8 and were well maintained at $47.47 \pm 7.75$ au and $57.78 \pm$ 9.23 au at week 12 , respectively. The stratum corneum hydration status of both groups increased over time, and significant differences were evident between the placebo group and GLA group ( $p=0.003$ ) (Fig. 4D).

The mean lipid concentration (sebumeter) did show nei- 
A

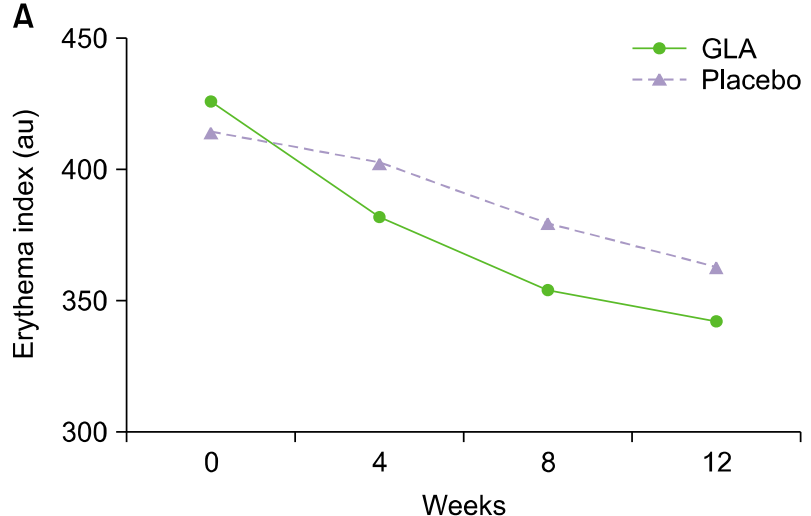

C
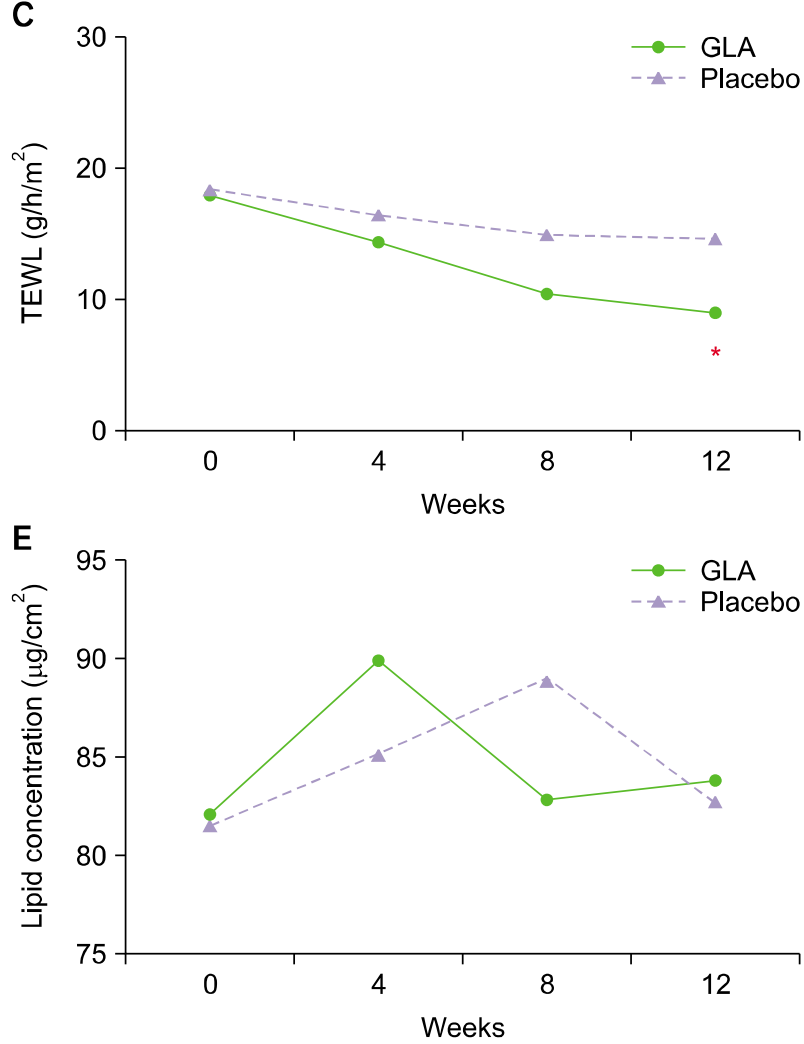

B

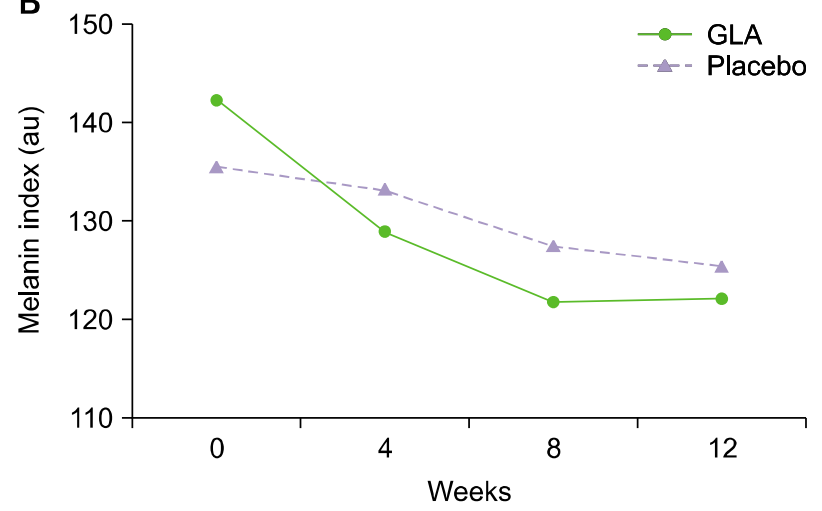

D

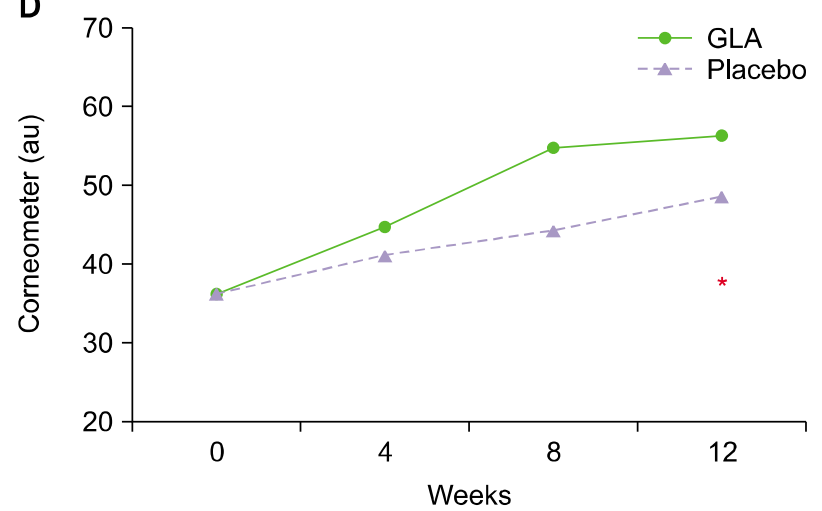

Fig. 4. Changes in biophysical parameters. (A) Erythema index, (B) melanin index, (C) transepidermal water loss (TEWL), (D) stratum corneum hydration, and (E) lipid concentration between placebo and gamma-linolenic acid (GLA) groups with rosacea. ${ }^{*} p<0.05$, repeated measures of analysis of variance.

ther consistent tendency nor significant difference between the placebo and GLA groups (Fig. 4E).

\section{Safety}

GLA was well generally well tolerated. One patient in the GLA group $(6.25 \%)$ reported diarrhea and one patient in placebo group $(6.67 \%)$ reported dyspepsia. However, the symptom resolved spontaneously within a few days without treatment. There was no serious AE.

\section{DISCUSSION}

Considering that the skin of rosacea patients is extremely sensitive and hyperreactive to dietary, environmental, and topical factors, systemic or topical agents can worsen stinging and dryness ${ }^{14}$. Therefore, additional treatment that can be sustained safely for a long period and can improve facial skin sensitivity is necessary for better treatment adherence and patient satisfaction.

In our study, significantly higher number of rosacea patients in the GLA groups achieved higher treatment suc- 
cess and reported higher satisfaction to treatment than those in the placebo group. At week 12, we observed $71.87 \%$ and $50 \%$ of IGA improvement, and $81.3 \%$ and $46.6 \%$ of PGA score 3 or 4 in the GLA and placebo groups, respectively. The erythema index and melanin index decreased in both GLA and placebo groups but did not differ significantly at the end of the treatment.

In this study, since GLA was taken with minocycline simultaneously, the overall improving tendency in the placebo group was thought to be an effect of minocycline. Minocycline has different actions with doxycycline in inhibition of matrix metalloproteinase, inhibition of bacterial products that stimulate inflammation, inhibition of granuloma formation, and suppression of neutrophil migration, and most effectively reduces reactive oxygen species ${ }^{15-18}$. A randomized controlled trial revealed that minocycline can be alternatively used for patients with PPR instead of doxycycline ${ }^{5}$. However, due to the chronic nature of rosacea, long-term treatment is often needed, which results in increased possibility of dose-related AEs such as hepatotoxicity ${ }^{6}$.

As far as we are aware, GLA supplementation has not previously been performed in rosacea patients. We observed that GLA supplementation as an add-on to minocycline had additional benefit in improving TEWL and stratum corneum hydration by restoring skin barrier function, which results in patient satisfaction.

In rosacea, chronic inflammation of the skin results in functionally impaired stratum corneum permeability barrier $^{4}$. Epidermal barrier defects can be demonstrated in a high proportion of rosacea patients compared with subjects who have normal skin ${ }^{4}$. Dirschka et al. ${ }^{19}$ reported decreased TEWL in the peri-nasal cheeks of rosacea patients. Steinhoff et al. ${ }^{20}$ demonstrated that the disruption of epidermal permeability barrier stimulates cytokine production, inflammatory cell infiltrate, LL-37 production, and vascular growth factor expression in rosacea. As a consequence, rosacea patients frequently experience symptoms such as dryness, stinging, and burning of the skin. In the National Rosacea Society Survey completed in 1997, 82\% of 1,023 patients complained of facial skin hyperirritability (sensitive skin) with burning, stinging, skin sensitivity, and skin irritation $^{21}$. These studies support that symptoms such as stinging, burning, and drying result from defective skin barrier.

There are few studies about biophysical parameters indicating skin barrier changes in the treatment of rosacea. Ní Raghallaigh and Powell ${ }^{22}$ reported that patients with PPR have increased erythema index, reduced epidermal hydration levels, and normal sebum levels, and treatment with systemic minocycline reduces erythema and increases hydration. This study corresponds with our baseline measurement data and improvement of biophysical profiles in the placebo group which received minocycline alone.

Notably, the TEWL decreased and stratum corneum hydration was increased significantly in the GLA group than in the placebo group. Topical and systemic GLA has been shown to regenerate a defective skin barrier in human skin $^{10}$. GLA can also increase ceramide synthesis, and GLA has an anti-inflammatory effect. GLA is metabolized into dihomo-gamma-linolenic acid (DGLA), which serves as a precursor of anti-inflammatory eicosanoids, such as prostaglandin E1 (PGE1) and 15-hydroxydihomo-r-linolenic acid (15-OH-DGLA). PGE1 inhibits proinflammatory cytokines, and 15-OH-DGLA inhibits 5-lipoxygenase, thereby reducing the production of arachidonic acid-derived proinflammatory eicosanoids ${ }^{12}$.

However, in this study, lipid concentration during the 12 weeks of treatment did not show any consistent tendency, which suggests that dietary supplementation of GLA does not affect skin surface lipid concentration. It can be explained from previous studies which demonstrated that neither lipid concentration nor excretion rate ${ }^{23}$ but the composition of sebaceous lipids is altered in rosacea patients compared with normal controls ${ }^{24}$.

This study has several limitations. First, our 8-week treatment duration is comparatively shorter than 12-week of other previous studies. Long-term data are still required to validate our result. Second, it was conducted in a single center with a relatively small sample size; thus, it could not reveal a difference in the additive effect of GLA between ETR and PPR. Therefore, future studies with larger number of patients and subgroup analysis between subtypes would further help the generalization of the results. We believe that our results add further data to demonstrate statistically significant and clinically meaningful improvements with GLA in rosacea symptoms of both ETR and PPR patients, accompanied by skin barrier restoration. In conclusion, we suggest that GLA is beneficial as an additional therapeutic option for rosacea patients treated with minocycline.

\section{CONFLICTS OF INTEREST}

The authors have nothing to disclose.

\section{FUNDING SOURCE}

This work was funded by Ulsan University Hospital (Biomedical Research Center Promotion Fund 17-06). 


\section{DATA SHARING STATEMENT}

Research data are not shared.

\section{ORCID}

Ji Hyun Kim, https://orcid.org/0000-0001-6784-7693

Yong Woo Oh, https://orcid.org/0000-0001-7169-7798

Dong Hee Kim, https://orcid.org/0000-0002-7727-1429

Byeong Hak Seo, https://orcid.org/0000-0002-7300-8236

Ho Seok Suh, https://orcid.org/0000-0002-6781-5429

Yu Sung Choi, https://orcid.org/0000-0001-8308-4091

\section{REFERENCES}

1. Crawford GH, Pelle MT, James WD. Rosacea: I. Etiology, pathogenesis, and subtype classification. J Am Acad Dermatol 2004;51:327-341; quiz 342-344.

2. Zhou M, Xie H, Cheng L, Li J. Clinical characteristics and epidermal barrier function of papulopustular rosacea: a comparison study with acne vulgaris. Pak J Med Sci 2016;32: 1344-1348.

3. Ahn CS, Huang WW. Rosacea pathogenesis. Dermatol Clin 2018;36:81-86.

4. Addor FA. Skin barrier in rosacea. An Bras Dermatol 2016; 91:59-63.

5. van der Linden $M M D$, van Ratingen $A R$, van Rappard DC, Nieuwenburg SA, Spuls PI. DOMINO, doxycycline $40 \mathrm{mg}$ vs. minocycline $100 \mathrm{mg}$ in the treatment of rosacea: a randomized, single-blinded, noninferiority trial, comparing efficacy and safety. Br J Dermatol 2017;176:1465-1474.

6. Harmon EG, McConnie R, Kesavan A. Minocycline-induced autoimmune hepatitis: a rare but important cause of druginduced autoimmune hepatitis. Pediatr Gastroenterol Hepatol Nutr 2018;21:347-350.

7. Wolfe ID, Reichmister J. Minocycline hyperpigmentation: skin, tooth, nail, and bone involvement. Cutis 1984;33:457458.

8. Laquieze S, Czernielewski J, Baltas E. Beneficial use of Cetaphil moisturizing cream as part of a daily skin care regimen for individuals with rosacea. J Dermatolog Treat 2007; 18:158-162.

9. Brosche T, Platt D. Effect of borage oil consumption on fatty acid metabolism, transepidermal water loss and skin parameters in elderly people. Arch Gerontol Geriatr 2000;30:
139-150.

10. Muggli R. Systemic evening primrose oil improves the biophysical skin parameters of healthy adults. Int J Cosmet Sci 2005;27:243-249.

11. Chung BY, Kim JH, Cho SI, Ahn IS, Kim HO, Park CW, et al. Dose-dependent effects of evening primrose oil in children and adolescents with atopic dermatitis. Ann Dermatol 2013;25:285-291.

12. Jung JY, Kwon HH, Hong JS, Yoon JY, Park MS, Jang MY, et al. Effect of dietary supplementation with omega-3 fatty acid and gamma-linolenic acid on acne vulgaris: a randomised, double-blind, controlled trial. Acta Derm Venereol 2014;94: 521-525.

13. Jeong MG, Kim JE, Ahn JY, Ko JY, Park MY, Ro YS. Effects of gamma-linolenic acid for the treatment of acne vulgaris treated with isotretinoin. Korean J Dermatol 2013;51:863-870.

14. Misery L. Sensitive skin and rosacea: nosologic framework. Ann Dermatol Venereol 2011;138(Suppl 3):S207-S210.

15. Alikhan A, Kurek L, Feldman SR. The role of tetracyclines in rosacea. Am J Clin Dermatol 2010;11:79-87.

16. Korting HC, Schöllmann C. Tetracycline actions relevant to rosacea treatment. Skin Pharmacol Physiol 2009;22:287-294.

17. Perret LJ, Tait CP. Non-antibiotic properties of tetracyclines and their clinical application in dermatology. Australas J Dermatol 2014;55:111-118.

18. Kraus RL, Pasieczny R, Lariosa-Willingham K, Turner MS, Jiang A, Trauger JW. Antioxidant properties of minocycline: neuroprotection in an oxidative stress assay and direct radical-scavenging activity. J Neurochem 2005;94:819-827.

19. Dirschka T, Tronnier H, Fölster-Holst R. Epithelial barrier function and atopic diathesis in rosacea and perioral dermatitis. Br J Dermatol 2004;150:1136-1141.

20. Steinhoff $M$, Buddenkotte J, Aubert J, Sulk M, Novak $P$, Schwab VD, et al. Clinical, cellular, and molecular aspects in the pathophysiology of rosacea. J Investig Dermatol Symp Proc 2011;15:2-11.

21. Torok HM. Rosacea skin care. Cutis 2000;66(4 Suppl):1416.

22. Ní Raghallaigh S, Powell FC. Epidermal hydration levels in patients with rosacea improve after minocycline therapy. $\mathrm{Br}$ J Dermatol 2014;171:259-266.

23. Burton JL, Pye RJ, Meyrick G, Shuster S. The sebum excretion rate in rosacea. Br J Dermatol 1975;92:541-543.

24. Ní Raghallaigh S, Bender K, Lacey N, Brennan L, Powell FC. The fatty acid profile of the skin surface lipid layer in papulopustular rosacea. Br J Dermatol 2012;166:279-287. 